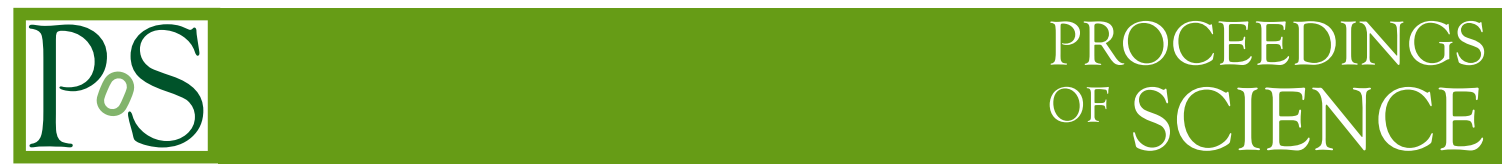

\title{
ALICE soft physics summary
}

\section{Dariusz Miśkowiec for the ALICE Collaboration}

GSI Darmstadt and CERN Geneva

E-mail: d.miskowiec@gsi.de

\begin{abstract}
Within the first two years of the LHC operation ALICE addressed the major soft physics observables in $\mathrm{Pb}-\mathrm{Pb}$ and $\mathrm{pp}$ collisions. In this contribution we present a selection of these results, with the emphasis on the bulk particle production and on particle correlations. The latter subject is discussed in detail in several dedicated ALICE talks in the same workshop; the reader is referred to the corresponding contributions.
\end{abstract}

The Seventh Workshop on Particle Correlations and Femtoscopy

September 20 - 242011

University of Tokyo, Japan 


\section{Introduction}

ALICE (A Large Ion Collider Experiment) is an experiment at the Large Hadron Collider dedicated to studies of QCD matter created in energetic collisions between lead nuclei [1]. QCD predicts that at energy densities above $1 \mathrm{GeV} / \mathrm{fm}^{3}$ a state of deconfined quarks and gluons occurs, possibly accompanied by chiral symmetry restoration in which quarks assume their current masses. Assessing the properties of the created matter requires sound understanding of the underlying collision dynamics which can be best studied via the bulk particle production observables. ALICE has a high granularity, a low transverse momentum threshold $p_{T}^{\min } \approx 0.15 \mathrm{GeV} / c$, and a good hadron identification up to several $\mathrm{GeV} / \mathrm{c}$ and is thus perfectly suitable for addressing soft physics observables in heavy-ion collisions. The setup is shown in Fig. 1. The central-barrel detectors, the Inner Tracking System (ITS), the Time Projection Chamber (TPC), the Transition Radiation Detector (TRD), and the Time Of Flight (TOF), cover the full azimuthal angle range at midrapidity $|\eta|<0.9$. The calorimeters EMCal and PHOS and the particle identification detector HMPID have partial coverage at midrapidity. The V0 detector measures charged-particle multiplicity at $-3.7<\eta<-1.7$ and $2.8<\eta<5.1$ and is mainly used for triggering and centrality determination. Several other detector systems exist but are not relevant to the results discussed in these proceedings. The collision systems and energies measured by ALICE are summarized in Table 1.

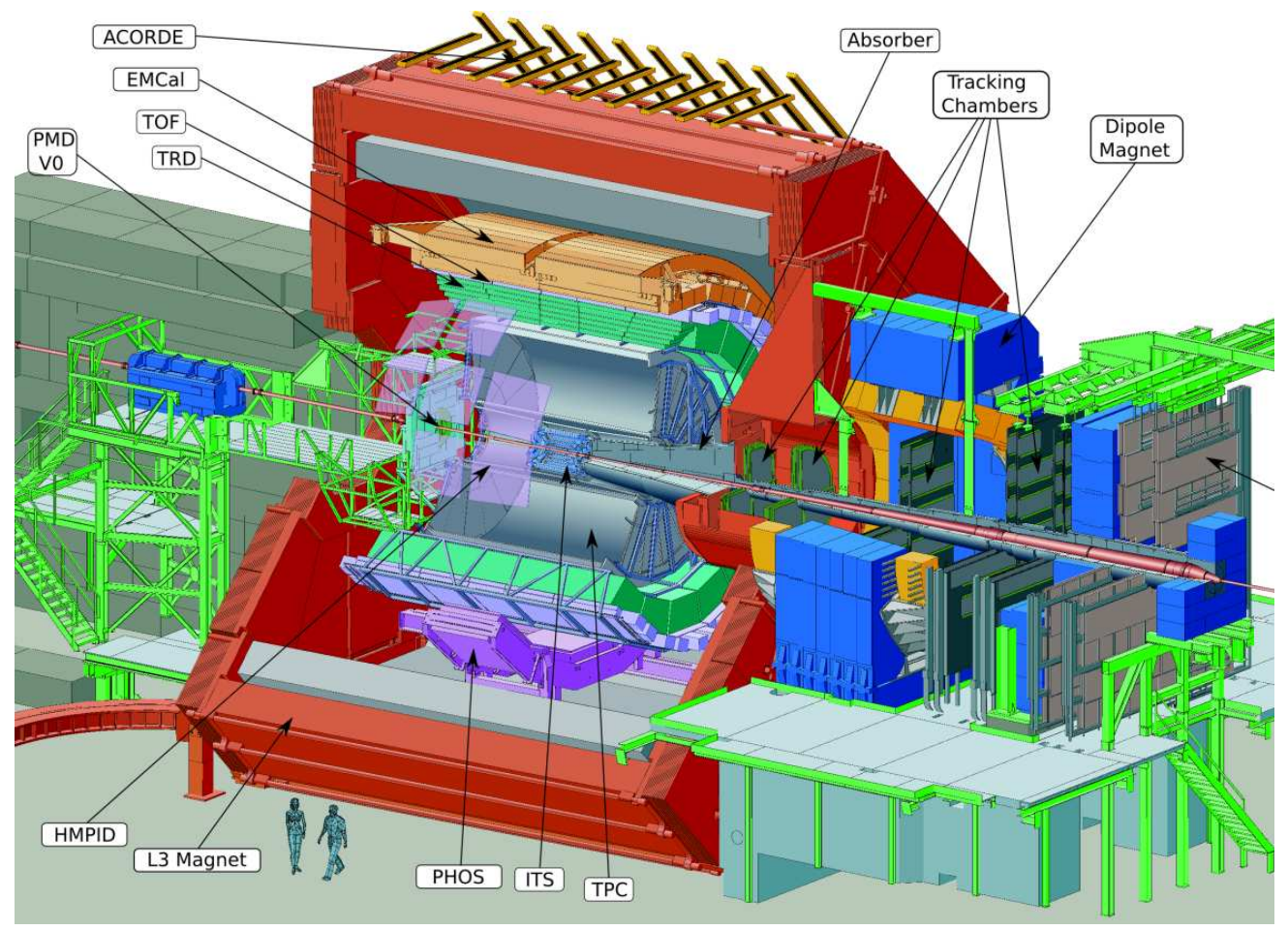

Figure 1: The ALICE experiment at the CERN LHC. The central-barrel detectors (ITS, TPC, TRD, TOF), with a pseudorapidity coverage $|\eta|<0.9$, address the particle production at midrapidity. The centrality is determined from the charged-particle multiplicity at $1.7<|\eta|<5.1$. 
Table 1: Data sets collected by ALICE in 2009-2012 (excluding rare triggers).

\begin{tabular}{lcccc}
\hline year & system & $\sqrt{s_{\mathrm{NN}}}(\mathrm{TeV})$ & trigger & \# events/10 \\
\hline 2009 & $\mathrm{pp}$ & 0.9 & min bias & 0.3 \\
2009 & $\mathrm{pp}$ & 2.36 & min bias & 0.04 \\
\hline 2010 & $\mathrm{pp}$ & 0.9 & min bias & 8 \\
2010 & $\mathrm{pp}$ & 7.0 & min bias & 800 \\
& & & high multiplicity & 50 \\
& & & forward muons & 50 \\
2010 & $\mathrm{~Pb}-\mathrm{Pb}$ & 2.76 & min bias & 30 \\
2010 & $\mathrm{pp}$ & 2.76 & min bias & 70 \\
\hline 2011 & $\mathrm{pp}$ & 7.0 & min bias & $\sim 10^{3}$ \\
2011 & $\mathrm{~Pb}-\mathrm{Pb}$ & 2.76 & central & $\sim 30$ \\
& & & semicentral & $\sim 30$ \\
& & & min bias & $\sim 10$ \\
& & & forward muons & $\sim 20$ \\
\hline
\end{tabular}

\section{Charged-particle production}

The bulk particle production is a basic indicator of entropy during a nuclear collision. The charged-particle pseudorapidity density in central $(0-5 \%) \mathrm{Pb}-\mathrm{Pb}$ collisions at $\sqrt{s_{\mathrm{NN}}}=2.76 \mathrm{TeV}$, normalized to the number of participant pairs, is $\mathrm{d} N_{\mathrm{ch}} / \mathrm{d} \eta /\left(0.5\left\langle N_{\text {part }}\right\rangle\right)=8.3 \pm 0.4$, about two times higher than in pp collisions at the same energy, and also about twice as high as in the goldgold collisions at RHIC (Fig. 2). Both in pp and $\mathrm{Pb}-\mathrm{Pb}$ the charged-particle pseudorapidity density is a power-law function of $\sqrt{s}$. The exponent is higher in nuclear collisions. The observed chargedparticle pseudorapidity density exceeds the predictions of most models with the initial state gluon saturation. The bulk particle production was subject of the first ALICE publication from the lead campaign at the LHC [2].

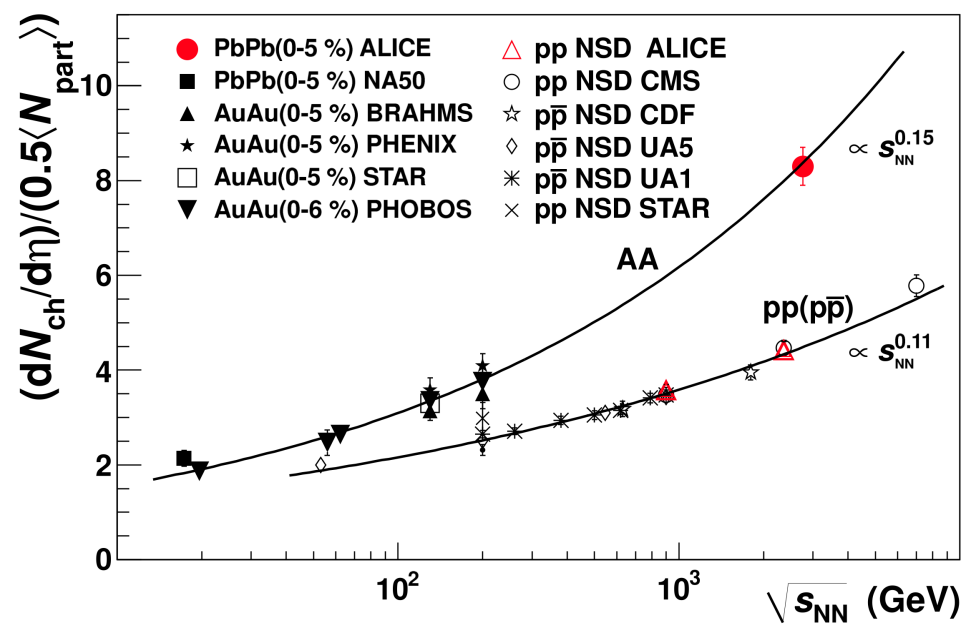

Figure 2: Charged-particle yields at midrapidity from pp and heavy-ion collisions [2]. 
More information is contained in the centrality dependence of the charged-particle pseudorapidity density. The centrality of the collision events, in terms of the fraction of the geometric cross section, was derived from the charged-particle multiplicity seen in the V0 detector at $1.7<|\eta|<5$.1. For this, the multiplicity distribution (Fig. 3) was fitted by a simple model assuming $f N_{\text {part }}+(1-f) N_{\text {coll }}$ particle sources, each source producing particles following a negative binomial distribution (red line). The number of participants and the number of binary collisions,

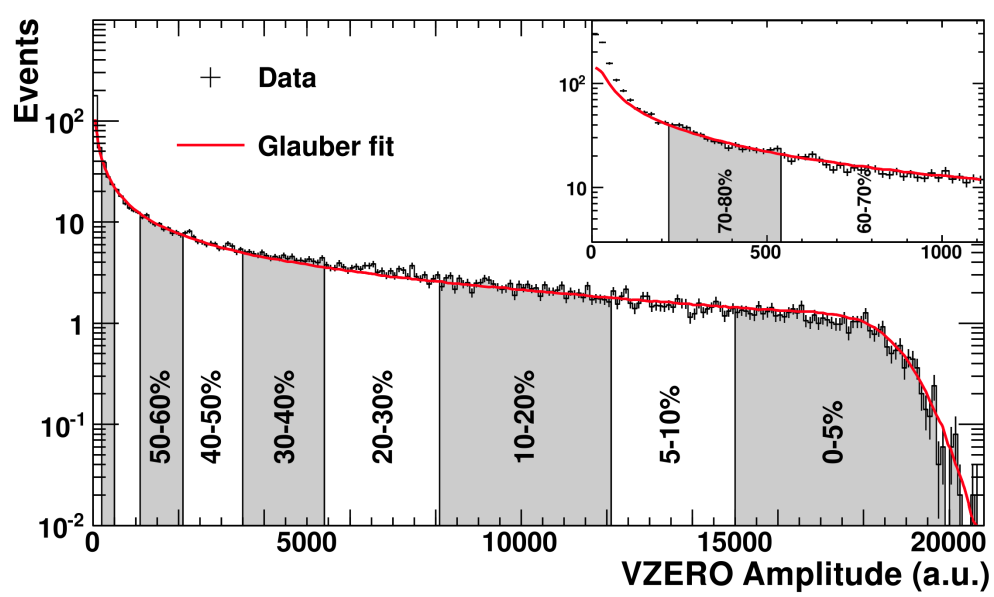

Figure 3: Determination of the centrality using the charged-particle multiplicity at $1.7<|\eta|<5.1$ [3].

$N_{\text {part }}$ and $N_{\text {coll }}$, were calculated for each impact parameter using Glauber model. The centrality resolution was better than $1 \%$. For the details of this analysis see Ref. [3].

The centrality dependence of the normalized charged-particle pseudorapidity density turns out to coincide in shape with the one measured at RHIC (Fig. 4). This is against the expectation that an increased contribution of hard processes should lead to a steeper centrality dependence at the LHC. The weak centrality dependence observed is presumably related to the nuclear shadowing and is, in fact, reasonably well reproduced by models which take this phenomenon into account [4].

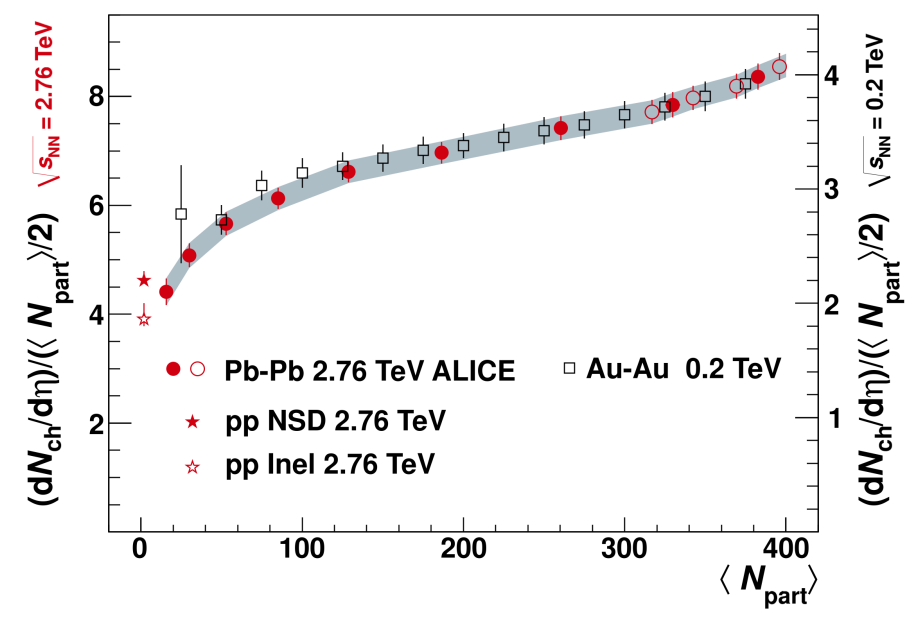

Figure 4: Charged-particle production as a function of centrality [3]. The LHC measurement coincides with the scaled RHIC data. 


\section{Identified hadrons}

A better handle on the reaction dynamics can be obtained using identified hadrons. Transverse momentum spectra of pions, kaons, and protons from $\mathrm{Pb}-\mathrm{Pb}$ collisions are shown in Fig. 5. The spectra at the LHC are harder than at RHIC [5]. A blast wave fit allows one to associate this fact with a $10 \%$ increase of the average transverse flow velocity.

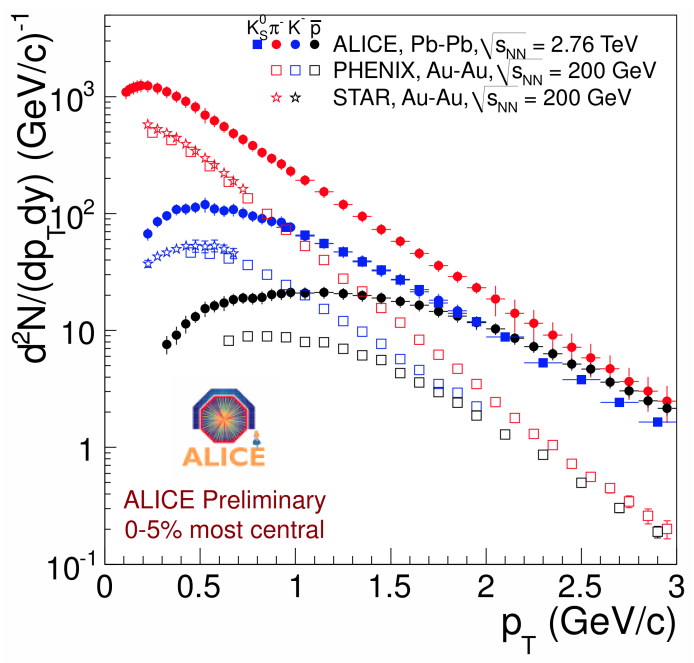

Figure 5: Transverse momentum spectra of identified hadrons from ALICE.

Hydrodynamic predictions [6] underestimate the high- $p_{T}$ part (Fig. 6). They also overestimate proton yield which might indicate a too high chemical freeze-out temperature $T_{\mathrm{ch}}$. A similar discrepancy is present in the thermal model. There, however, a lower $T_{\mathrm{ch}}$ is excluded by the $\Xi$ and $\Omega$ yields [7]. The details of the identified hadron analysis are given in Ref. [5].

Another exciting prospect is to look for production of light nuclei and hypernuclei. Combining the specific energy loss in the TPC with the time of flight from TOF, ALICE was able to identify four anti-alpha particles in the $2010 \mathrm{~Pb}-\mathrm{Pb}$ data set. An order of magnitude more abundant was
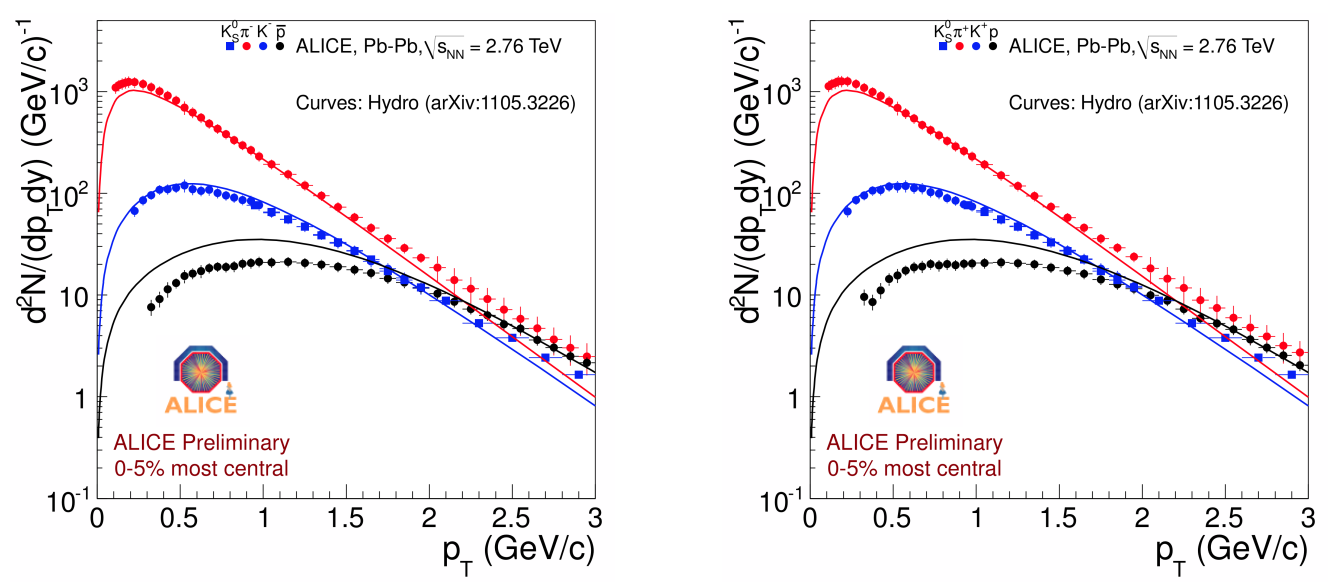

Figure 6: Transverse momentum spectra of identified hadrons compared to hydro predictions. 
the antihypertriton $\frac{3}{\Lambda} \overline{\mathrm{H}}$ detected via its decay into ${ }^{3} \overline{\mathrm{He}}$ and $\pi^{+}$. For antihelium ${ }^{3} \overline{\mathrm{He}}$, a transverse momentum spectrum was measured in the range of 1-8 GeV/c. The search for such composed objects with ALICE is discussed in Refs. $[8,9,10]$.

\section{Femtoscopy}

An increase of the spatial extension of the particle source from RHIC to LHC energies was declared a decisive test of whether the concept of "matter" is at all applicable to the system created in energetic nuclear collisions [11]. Spatial extension of the source of pions with a fixed momentum vector (homogeneity length, or Hanbury Brown-Twiss (HBT) radius) is accessible via the BoseEinstein correlations aka HBT analysis. The pion homogeneity volume measured by ALICE in most central 5\% Pb-Pb collisions at $\sqrt{s_{\mathrm{NN}}}=2.76 \mathrm{TeV}$ [12] is shown in Fig. 7. The volume is about two times larger than the analogous volume at RHIC and scales linearly with multiplicity.

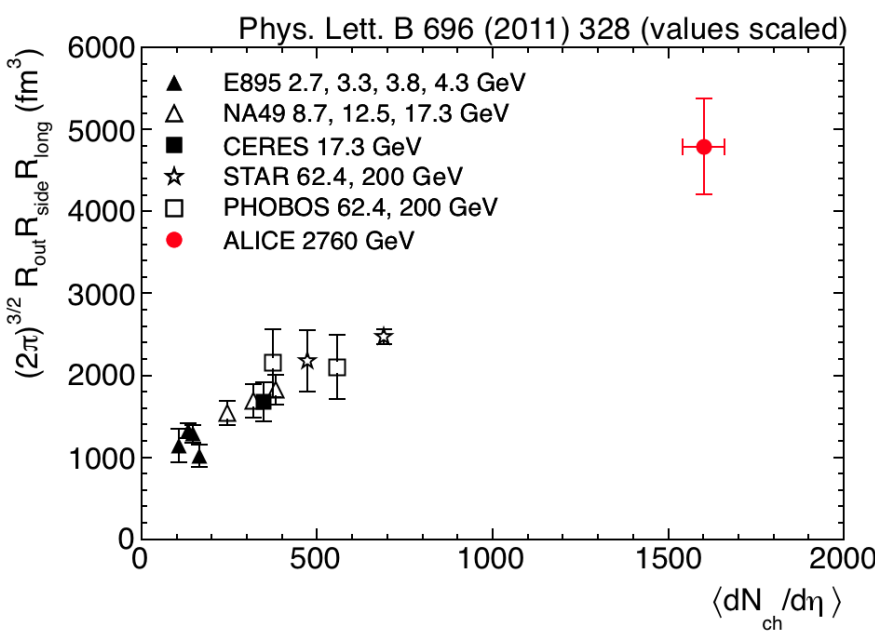

Figure 7: Homogeneity volume in central gold and lead collisions at different energies [12].

While the homogeneity volume of central heavy-ion collisions observed at various collision energies nicely scales with the charged-particle pseudorapidity density, including other centralities and, in particular, other collision systems leads to a violation of this scaling. This is particularly clear when comparing the volumes measured at the LHC in $\mathrm{pp}$ and $\mathrm{Pb}-\mathrm{Pb}$ at the same chargedparticle pseudorapidity density (Fig. 8) [13, 14]. The three HBT radii always scale linearly with the cube root of $\left\langle\mathrm{d} N_{\mathrm{ch}} / \mathrm{d} \eta\right\rangle$ but the slope of the scaling is different for protons and heavy-ions. This indicates that the HBT radii are not driven exclusively by the final multiplicity but are also sensitive to the initial geometry of the collision.

The collective outward motion of particles at freeze-out is the commonly accepted explanation for the fact that the HBT radii in heavy-ion collisions decrease with increasing transverse momentum. The observation of a similar $p_{T}$ dependence in pp collisions at RHIC suggested that either this explanation has to be revised, or collective flow exists also in pp collisions. Some clarification on the subject and support to the latter statement comes from the observation that the $p_{T}$ dependence in pp collisions develops with increasing multiplicity $[15,13]$. This is shown in Fig. 9 and discussed in detail in Refs. [13, 14, 16]. 

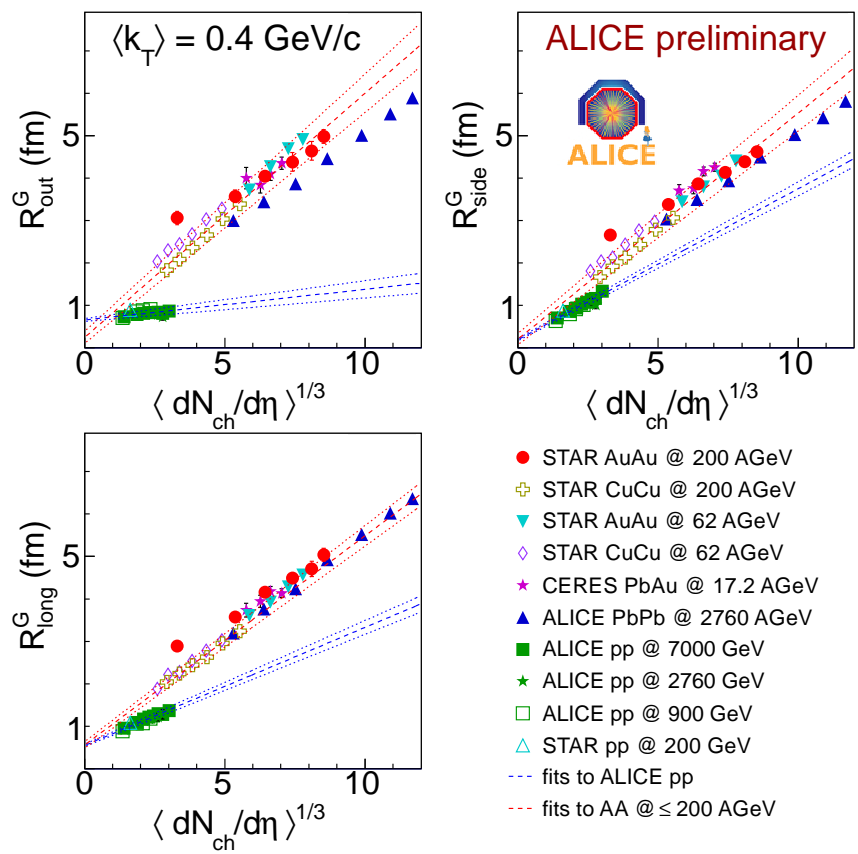

- STARAuAu @ 200 AGeV

STAR CuCu@200 AGeV

STARAuAu @ 62 AGeV

STAR CuCu @ 62 AGeV

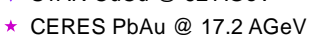

\ ALICE PbPb @ 2760 AGeV

— ALICE pp@ $9000 \mathrm{GeV}$

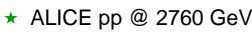

$\square$ ALICE pp @ $900 \mathrm{GeV}$

$\triangle$ STAR pp @ $200 \mathrm{GeV}$

- fits to ALICE pp

- fits to AA @ $\leq 200$ AGeV

Figure 8: Scaling of HBT radii with the charged-particle pseudorapidity density in pp and AA collisions.

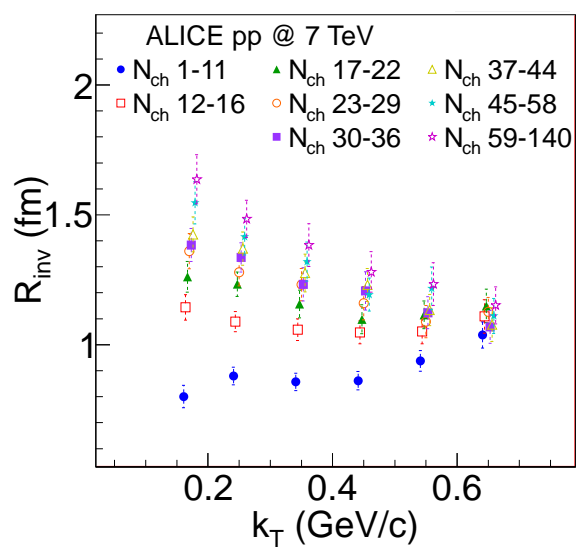

Figure 9: Evolution of the transverse momentum dependence of HBT radii with multiplicity in pp collisions [13].

\section{Elliptic flow}

After the RHIC experiments found that the QCD matter created in heavy-ion collisions behaved like a low-viscosity fluid, a natural question arose whether this behavior would continue at higher collision energies, or whether the system would get closer to a non-interacting gas of quarks and gluons. This question was answered by the ALICE measurement of the elliptic flow coefficient $v_{2}$ which quantifies the azimuthal anisotropy of the particle emission in non-central collisions and which, therefore, is sensitive to the early stage of the collision [17]. As is shown in Fig. 10, the elliptic flow at the LHC turned out to be higher than at RHIC and to follow the trend observed at lower energies. The $v_{2}$ increase at the LHC mostly comes from the increase in $\left\langle p_{T}\right\rangle$, as the shape 


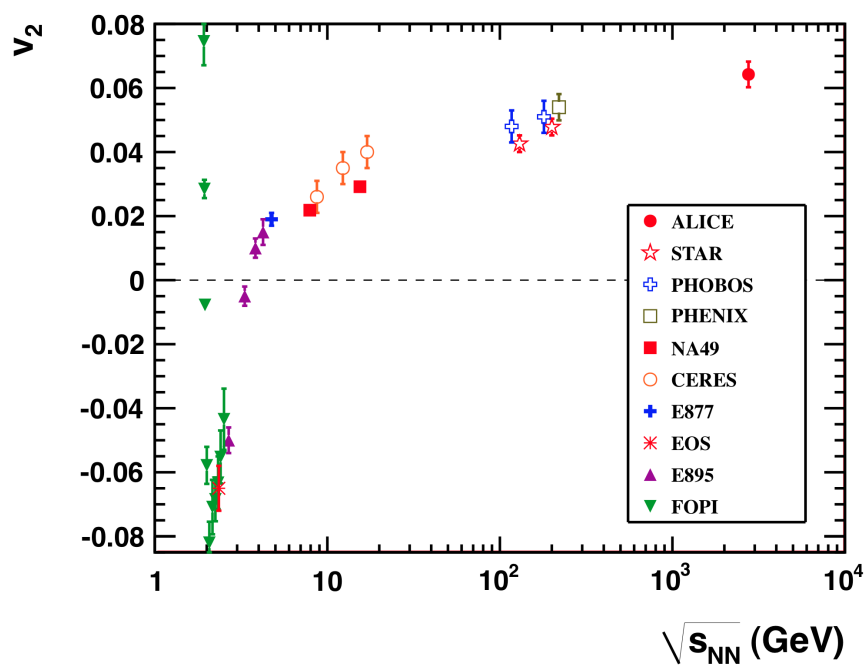

Figure 10: Collision energy dependence of the elliptic flow [17]. The ALICE measurement matches the trend established at lower energies.

of the $v_{2}\left(p_{T}\right)$ dependence remains unchanged within the $\sqrt{s_{\mathrm{NN}}}$ range from 40 to $2760 \mathrm{GeV}$.

The elliptic flow coefficient of identified hadrons shows a splitting characteristic to the presence of transverse radial flow (Fig. 11) $[18,19]$. The shape of the $p_{T}$ dependence and the presence of the splitting are fairly well reproduced by hydrodynamics [20]. The calculation, however, underpredicts the elliptic flow of protons. This can be cured by adding hadronic rescattering [20].

An important role at the LHC is played by the triangular flow. Its coefficient $v_{3}$ only weakly depends on centrality and its direction is not related to the orientation of the event plane which points to the initial energy density fluctuations as its origin. There are indications that the "Mach cone"-like azimuthal emission pattern is actually just a superposition of the elliptic and triangular collective flow $[21,22,19]$.

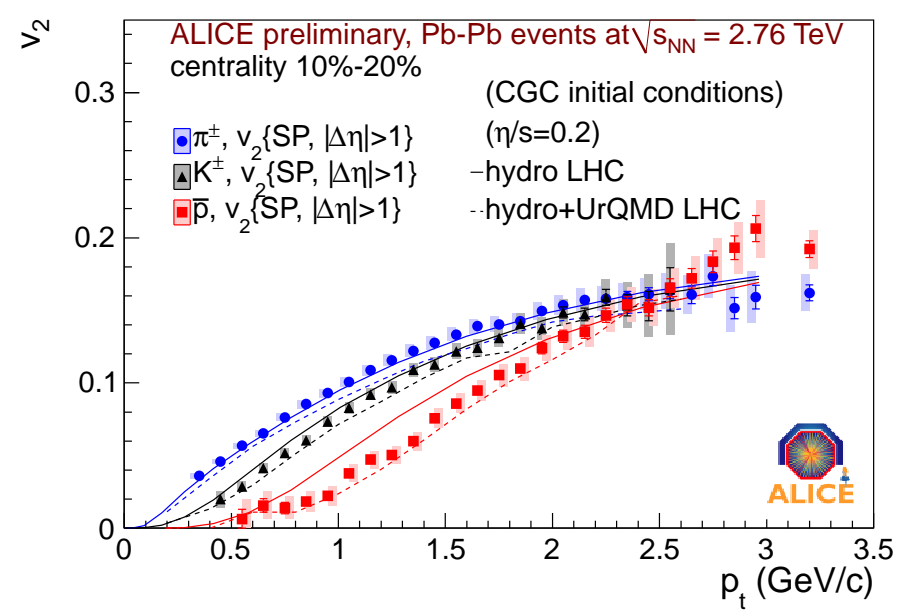

Figure 11: Transverse momentum dependence of the elliptic flow of pions, kaons, and protons. The splitting is presumably caused by transverse radial flow. 


\section{Fluctuations}

The magnitude of charge fluctuations should reflect the number of charge carriers, and thus be different for QGP and hadron gas. It is puzzling that the experimental results so far were closer to the hadron gas calculation than to QGP (although the distinction is somewhat obscured by resonances that introduce anticorrelations between positive and negative charges). In an ALICE analysis of $\mathrm{Pb}-\mathrm{Pb}$ at $\sqrt{s_{\mathrm{NN}}}=2.76 \mathrm{TeV}$ the net-charge fluctuations, expressed in terms of $v_{(+,-, \text {dyn })}=$ $\left\langle\left(\frac{N_{+}}{\left\langle N_{+}\right\rangle}-\frac{N_{-}}{\left\langle N_{-}\right\rangle}\right)^{2}\right\rangle-\frac{1}{\left\langle N_{+}\right\rangle}-\frac{1}{\left\langle N_{-}\right\rangle}$and corrected for the finite acceptance, approach the QGP value in most central collisions as shown in the left panel of Fig. 12 (see Ref. [23] for details).

Enhanced transverse momentum fluctuations may signal vicinity to the critical point of the QCD phase transition. The ALICE measurement of the $p_{T}$ fluctuations, expressed via the mean covariance between transverse momenta of track pairs $C_{m}$, is shown for $\mathrm{Pb}-\mathrm{Pb}$ and pp collisions in the right hand panel of Fig. 12. Unlike the HBT radii discussed above, the relative $p_{T}$ fluctuations fall on a universal curve when plotted against multiplicity. The dependence is of the power-law type, except for deviations at very soft $\mathrm{pp}$ and at central $\mathrm{Pb}-\mathrm{Pb}$ collisions. The latter is not reproduced by the HIJING event generator. The details of this analysis are given in Ref. [24].
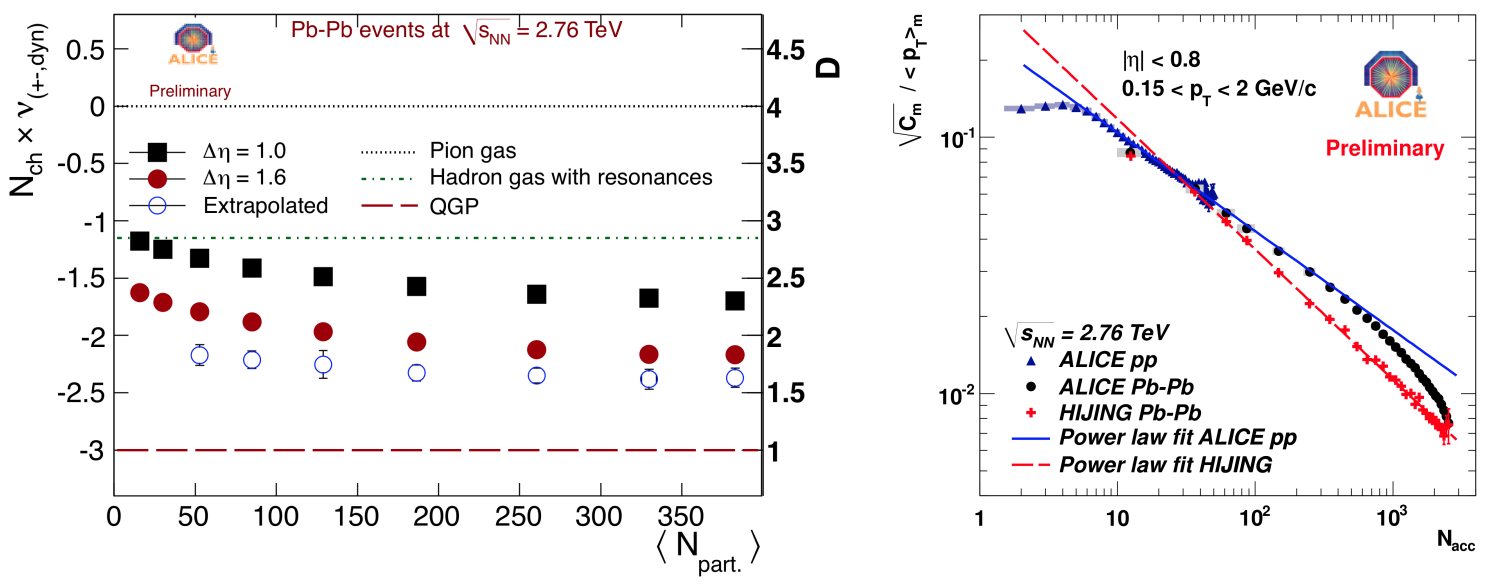

Figure 12: Multiplicity dependence of charge (left) and $p_{T}$ (right) fluctuations measured by ALICE.

The transient magnetic field of two ions colliding at a finite impact parameter may lead to charge-dependent angular correlations. The STAR experiment at RHIC reported such correlations developing when going from central to peripheral collisions of gold nuclei [25]. The analogous measurement in $\mathrm{Pb}-\mathrm{Pb}$ performed by ALICE agrees within errors with the STAR result [26].

\section{Summary}

During the first campaign of $\mathrm{Pb}-\mathrm{Pb}$ collisions at the LHC, ALICE addressed the most important soft physics observables. The lead collision studies were augmented by pp measurements at several energies. New insights into the reaction dynamics include an alternative interpretation of the angular emission pattern (flow rather than the "Mach cone"), the $p_{T}$ dependence of HBT radii developing with multiplicity in pp collisions, and the proton puzzle (lower than expected yield and elliptic flow). 
As the c.m.s. energy increase from RHIC to LHC is unprecedentedly large it is interesting to verify whether the energy dependence trends found at lower energies are still valid at the LHC. The results discussed in this paper fall in three categories. First, about two times larger than at RHIC are the particle yield and the homogeneity volume. Second, an increase by $10-30 \%$ is observed in the transverse flow, mean transverse momentum, integrated elliptic flow, and the mass splitting of $v_{2}$. (The latter three may be a consequence of the first.) Finally, unchanged with respect to RHIC remained the centrality dependencies of the particle yield and of $v_{2}$, the multiplicity dependencies of pion HBT radii and of particle ratios, the $p_{T}$ dependence of $v_{2}$, and the charge and $p_{T}$ fluctuations.

The author thanks the organizers for the pleasant and inspiring atmosphere during the meeting.

\section{References}

[1] F. Carminati, (Ed.) et al. [ALICE Collaboration], J. Phys. G G 30 (2004) 1517; G. Alessandro, (Ed.) et al. [ALICE Collaboration], J. Phys. G G 32 (2006) 1295.

[2] B. Abelev et al. [The ALICE Collaboration], Phys. Rev. Lett. 105 (2010) 252301 [arXiv:1011.3916].

[3] K. Aamodt et al. [ALICE Collaboration], Phys. Rev. Lett. 106 (2011) 032301 [arXiv:1012.1657].

[4] B. Muller, J. Schukraft and B. Wyslouch, arXiv:1202.3233 [hep-ex].

[5] M. Floris, J. Phys. G G 38 (2011) 124025 [arXiv:1108.3257].

[6] C. Shen, U. Heinz, P. Huovinen and H. Song, Phys. Rev. C 84 (2011) 044903. [arXiv:1105.3226].

[7] A. Kalweit, Proceedings of Strange Quark Matter 2011, to be published in Acta Phys. Polon.

[8] A. Kalweit [ALICE Collaboration], J. Phys. G G 38 (2011) 124073 [arXiv:1107.1514].

[9] N. Sharma [ALICE Collaboration], [arXiv:1104.3311].

[10] R. Lea, Proceedings of Strange Quark Matter 2011, to be published in Acta Phys. Polon.

[11] H. Satz, Nucl. Phys. A 862-863 (2011) 4 [arXiv:1101.3937].

[12] K. Aamodt et al. [ALICE Collaboration], Phys. Lett. B 696 (2011) 328 [arXiv:1012.4035].

[13] K. Aamodt et al. [ALICE Collaboration], Phys. Rev., D84 (2011) 112004 [arXiv:1101.3665].

[14] A. Kisiel, these proceedings; $Ł$. Graczykowski, these proceedings.

[15] V. Khachatryan et al. [CMS Collaboration], JHEP 1105 (2011) 029 [arXiv:1101.3518].

[16] T. Humanic, these proceedings.

[17] K. Aamodt et al. [The ALICE Collaboration], Phys. Rev. Lett. 105 (2010) 252302 [arXiv:1011.3914].

[18] F. Noferini, Proceedings of Strange Quark Matter 2011, to be published in Acta Phys. Polon.

[19] C. Ivan, these proceedings.

[20] U. W. Heinz, C. Shen and H. Song, [arXiv:1108.5323].

[21] K. Aamodt et al. [ALICE Collaboration], Phys. Rev. Lett. 107 (2011) 032301 [arXiv:1105.3865].

[22] M. Sano, these proceedings.

[23] S. Jena, these proceedings.

[24] S. Heckel [ALICE Collaboration], J. Phys. G 38 (2011) 124095 [arXiv:1107.4327].

[25] B. I. Abelev et al. [STAR Collaboration], Phys. Rev. Lett. 103 (2009) 251601 [arXiv:0909.1739].

[26] I. Selyuzhenkov, these proceedings. 